\title{
Vi sono ragioni della libertà note al cuore e all'intelletto: il triplo sguardo sulla libertà espresso nei Ritiri spirituali di Parigi di Józef Tischner
}

\section{There are motives of freedom, known to man's heart and mind - a threefold look at freedom in Józef Tischner's "Rekolekcje Paryskie"}

"Quando siamo noi stessi ci sentiamo a casa nostra. Se ci sentiamo a casa nostra siamo noi stessi. La via che porta a questa condizione è l'amicizia con Dio" ${ }^{1}$. 
and theological perspective. Tischner proposes no definitive interpretation of freedom but, viewing the controversy over freedom as one of the most difficult problems in the philosophy of man, he tries to show what man's inner freedom consists in and what achievements it makes man capable of.

\section{Keywords}

Freedom, heart, soul, drama.

Se la libertà è una questione che riguarda ogni uomo e costituisce uno dei problemi filosofici più spesso analizzati, nel pensiero filosofico-etico-sociale di Józef Tischner esso occupa un posto centrale. Tutto ciò che riguarda l'individuo e i suoi rapporti con il prossimo si realizza attraverso la libertà e grazie ad essa. La libertà è come una sorgente nello spazio degli umani riferimenti, ma questa sorgente è indissolubilmente legata con il dramma che abbraccia tutta la realtà etica e assiologica dell'uomo. Alla libertà partecipa l'uomo in tutta la sua interezza e il radicarsi della libertà sempre più a fondo nell'uomo avviene attraverso la realizzazione di scelte morali e la decisione di praticare il bene.

L'uomo esige la libertà, ne soffre la mancanza e per essa a volte lotta. Per la libertà l'uomo è pronto a sacrificarsi, disponendo di essa nel modo più consono alla propria natura. A seconda del modo in cui l'uomo fruisca della propria libertà e verso quali valori egli orienti le proprie aspirazioni, è in grado di pervenire alla pienezza dell'umanità "vincendo" se stesso oppure, scegliendo il male, svalutare ciò che in lui vi è di umano, fino a "perdere" se stesso.

Józef Tischner ha sottolineato spesso che il dissidio vertente sulla libertà rientra fra le questioni più serie e più importanti affrontate dalla filosofia dell'uomo nonché tra le sfide più difficili dell'età contemporanea. Ha segnalato inoltre che è possibile perseguire due vie filosofiche per arrivare a capire cosa sia la libertà e darne una definizione: la via dell'ontologia e la via della filosofia del dramma. Entrambe mostrano tutto ciò che della libertà è costitutivo e fondamentale, ma la strada della filosofia del dramma è stata per Tischner l'unica in grado di accedere all'area delle relazioni con l'Altro, anche attraverso lottica espressa dalla domanda: "che tipo di legame unisce la persona umana con la persona di Dio?"2.

${ }^{2}$ J. Tischner, Zarys filozofii człowieka dla duszpasterzy i artystów [Profilo di filosofia dell'uomo per sacerdoti e artisti], in: „Myślenie w żywiole piękna”, Znak, Kraków 2004, p. 156. 
Nel suo lascito pubblicistico, scientifico e didattico, Tischner ha lasciato ai posteri numerose dichiarazioni e riflessioni testuali sulla libertà, la cui cura redazione, sistemazione e caratterizzazione richiedono ricerche complesse e articolate su più livelli. Per tale ragione nell'ambito del presente studio si tratterà solamente di alcune intuizioni del filosofo che non sono certamente in grado di svelare il senso più stretto del concetto di libertà, ma possono illuminare almeno parzialmente la ben più ampia prospettiva filosofica di Tischner sulla libertà. Saranno esposte alcune questioni filosofiche tratte dai Ritiri spirituali di Parigi (conferenze tenute negli anni '70 del secolo scorso rivolte agli emigrati polacchi residenti a Parigi, non pubblicate fino al 2013), che negli anni a seguire avrebbero trovato espressione in analisi più precise raccolte in varie opere di Tischner, tra le quali Myślenie w żywiole piękna [Pensare nellelemento del bello], Filozofia dramatu [Filosofia del dramma], Spór o istnienie człowieka [Controversia sullesistenza dell'uomo], Nieszczęsny dar wolności [Lo sfortunato dono della libertà], Świat ludzkiej nadziei [Il mondo della speranza umana], nonché in molti articoli scientifici.

\section{La libertà come essere scelti}

Nel capitolo intitolato La fede nasce dalla scelta dei Ritiri spirituali di Parigi Tischner analizza il fenomeno non soltanto della scelta in sé, ma anche dell'essere scelti. Ė proprio grazie a questo fenomeno che la libertà ha la possibilità di manifestarsi. Nell'essere scelti non è importante tanto chi ci abbia scelti (la madre, il padre, Dio, chi ci ama), quanto invece il fatto che tale persona abbia compiuto una scelta. Alla base di ogni relazione giace la scelta e fino a quando non si pervenga ad una scelta reciproca, resta impossibile capire fino in fondo sé stessi e tanto meno la libertà che instaura le relazioni. Scrive Tischner: "Luomo non capisce la libertà finché non venga scelto da altri”" ${ }^{3}$. Il fatto che io venga scelto da qualcuno è esso stesso una conferma del sussistere della libertà, ma costituisce anche una testimonianza del fatto che per compimento della libertà del prossimo si diventa, per tale individuo, una persona importante in quanto scelta fra altre. È probabile che l'atto dello scegliere si riveli appieno nellatto d'amore, in cui due individui scegliendosi reciprocamente si sono dati e affidati l'un l'altro. Prima ancora di scegliersi, tuttavia, essi hanno dovuto "riconoscere"

3 J. Tischner, Rekolekcje Paryskie, Znak, Kraków 2013, p. 67. 
ciò che avrebbero scelto ed effettuare l'atto di rispondere alla scelta. Ma anche dare una risposta significa scegliere. La risposta all'essere scelti diviene come il momento nodale della responsabilità poiché nello scegliere si è compiuto un rivolgimento e un rivolgersi verso l'altro, che a partire da tale momento non potrà più essere tradito. Il legame della fedeltà nasce nel momento in cui viene accettata la scelta compiuta dall'altro. Essere fedeli significa "portare in se stessi la scelta compiuta. [...] La fedeltà consiste nel fatto che in ogni momento è necessario compiere una scelta"4.

Fino al momento in cui si viene scelti, la libertà è semplicemente come un banco di numerose possibilità, è potenzialità non ancora mutata in scelta. Tuttavia nel momento in cui viene effettuata una scelta è come se la libertà raggiungesse se stessa ritrovandosi in sé. Nel compiere una scelta un ampio ventaglio di possibilità muta in un'unica scelta e quest'unica scelta è come una conclusione, un riempimento, chiarezza. Inoltre, attraverso la scelta giunge a manifestazione un senso specifico di ciò che si è scelto. "La libertà consiste nel fatto che io posso scegliere colui che ha scelto me. Scelta per scelta, nome per nome"s.

Laddove non cè scelta, non hanno modo di esistere né l'amore, né la fede. Ma non vi può essere fedeltà neppure laddove manchi la possibilità costante di scegliere. A tal proposito le parole di Tischner suonano drammaticamente: "se l'uomo dovesse decidere di non portare Dio sulle spalle della propria libertà,

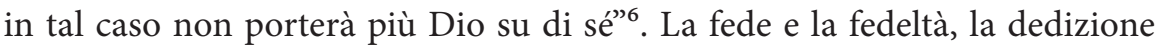
e l'amore hanno origine dalla scelta. Bisogna prima scegliere, ma facendolo per nome, perché "chiunque parli per mezzo di un nome, parla anche in nome della

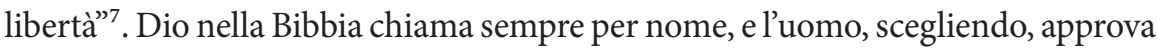
il prossimo come un individuo che possiede un nome proprio. Anche l'uomo riconosce se stesso come persona scelta, recante un nome proprio. La libertà, precedentemente soltanto intuita, nell'istante della scelta per nome diviene viva esperienza, autentica realtà. L'uomo, nel momento in cui vive l'esperienza di essere scelto, sente nascere una nuova qualità, sente che tutto acquista un senso

4 Testo inedito. La citazione è tratta da un'incisione audio provvista di segnatura NA/91-93 del discorso tenuto da J. Tischner presso la Chiesa delle SS. Clarisse di Stary Sącz ed è proprietà dell'Istituto del Pensiero di Józef Tischner di Cracovia.

5 J. Tischner, Rekolekcje Paryskie, Znak, Kraków 2013, p. 68.

${ }^{6}$ J. Tischner, Rekolekcje Paryskie, Znak, Kraków 2013, p. 70.

7 J. Tischner, Rekolekcje Paryskie, Znak, Kraków 2013, p. 67. 
nuovo e che egli stesso, a partire da tale momento ha un significato e un senso per qualcuno. Ogni cosa assume un senso: l'identità dell'Io e la sua relazione con il $\mathrm{Tu}$, ma anche la realtà compresa fra l'Io e il Tu.

Tischner lega la scelta all'amore. L'amore è una scelta. Chi è scelto e a sua volta può effettuare la scelta di scegliere ama tanto più, quanto più sceglie la persona amata. Nella relazione tra uomo e Dio si sceglie Colui che ha già scelto noi e che quindi già ci amava. Tischner afferma che quanto più maturo è l'amore, tanto più matura è la scelta, ma il filosofo non afferma la situazione opposta, in cui è l'amore a presupporre la maturità della scelta, e pertanto l'opportuna qualità, contenuto e valore della medesima. Sembra che quanto meno matura sia la scelta, tanto meno maturo sarà, necessariamente, l'amore. La relazione tra amore e libertà deve essere completata dalla reciprocità della libertà matura e dell'amore maturo. Come tuttavia sia possibile conseguire l'amore e la libertà maturi, quali criteri e condizioni li garantiscano è espresso da Tischner a grandi tratti. Il filosofo, nei Ritiri spirituali di Parigi, affronta i problemi dell'umiltà, del pentimento, della gratitudine come aspetti responsabili dello sviluppo nell'uomo di questo tipo di maturità. È lecito dichiarare che Tischner abbia individuato la tendenza (o fine) dell'aspirazione dell'uomo all'amore e la tendenza della realizzazione dell'uomo alla libertà. Anche per tale motivo la fede come legame tra persone libere viene conseguita grazie alla libertà e attraverso di essa. La fede nasce dalla scelta e nella scelta definisce se stessa, si chiarifica, divenendo una relazione con Dio. Tischner constata: "Finché non vi sarà Dio tra gli uomini, non esisterà religione. Ma Dio non sarà tra gli uomini se non saranno loro a scegliere Dio attraverso la fedeltà, attraverso la scelta, attraverso il nome, attraverso la libertà"8.

\section{La libertà come spazio}

Nel capitolo intitolato Lo spazio della nostra libertà (omelia del 3 marzo 1985) Tischner fa riferimento a due generi di spazio: lo spazio esterno del mondo e lo spazio interiore dell'anima. Entrambi questi spazi si compenetrano, si intrecciano e si sovrappongono. In entrambi ha luogo una diversificazione quantitativa e qualitativa. Nello spazio esterno del mondo Tischner riconosce un ordine laddove ogni cosa ha un suo luogo, un suo status, svolge un determinato

8 J. Tischner, Rekolekcje Paryskie, Znak, Kraków 2013, p. 71. 
ruolo. In questo spazio ogni cosa partecipa ad un certo ordine, in cui ogni determinata cosa "non ha bisogno di lottare con un'altra cosa, non è in conflitto con essa, non deve distruggere un'altra cosa per poter esistere" . Invece lo spazio interiore dell'uomo è situato nei recessi dell'anima umana e qui trova la propria espressione. Proprio questo genere di spazio interessa maggiormente a Tischner. Egli osserva che la diversificazione delle persone a livello spirituale ha le sue radici nella libertà.

È possibile parlare di due "direzioni" alle quali tenda l'anima umana a seconda del contrarsi e dell'estendersi in essa dello spazio della libertà. Vi sono infatti individui che attraverso atti di libertà è come se restringessero lo spazio del proprio spirito ("anima stretta"), ma vi sono anche quanti non facciano che ampliare costantemente tale spazio ("anima larga") e svilupparlo ("anima profonda"). Vi sono anche quanti nello spazio attuale dell'anima non si accorgono del suo progressivo restringersi ("anima piatta"). Che lo spazio interiore dell'uomo non si disintegri dipende solamente dall'uomo e dalla sua responsabilità per la propria interiorità. Grazie alla libertà, infatti, possiamo ampliare tale spazio o recuperare lo spazio perduto, o anche intraprendere una via diretta ad uno spazio che si estende dinnanzi a noi.

Tischner segnala che la libertà è uno spazio sui generis in grado a sua volta di dare origine ad uno spazio ancora più grande: la libertà stessa è spaziosa. Essa racchiude tutto quanto vi sia nell'uomo di ragionevole e irragionevole, responsabile e irresponsabile, piatto e profondo, creativo e caotico. La libertà giace alla base di tutto questo. Tischner sottolinea che "chi è libero ha uno spazio, ma chi è costretto è come racchiuso in uno spazio ristretto, e questa ristrettezza è situata nella sua stessa anima" ${ }^{10}$. Più lo spazio interiore dell'anima viene approfondito e sviluppato, tanto più essa capisce dello spazio esterno del mondo; e al contrario, tanto più l'uomo rifiuta la libertà, rifugge da essa o la teme, quanto più la visione dello spazio esterno è falsata. L'uomo diviene allora ostaggio della sua stessa libertà, la sua anima diviene via via più "stretta". Tischner scrive che "quando dall'anima dell'uomo si estingue il sentimento della libertà, il mondo attorno a lui gli diviene estraneo, nemico, angusto. [...] Quest'uomo costruisce una torre nella propria anima, si costringe ad essa con una catena, mentre questa torre gli copre il cielo e gli sottrae la libertà"11. Tale "ristrettezza dell'anima"

\footnotetext{
9 J. Tischner, Rekolekcje Paryskie, Znak, Kraków 2013, p. 85.

10 J. Tischner, Rekolekcje Paryskie, Znak, Kraków 2013, p. 86.

11 J. Tischner, Rekolekcje Paryskie, Znak, Kraków 2013, p. 86.
} 
è una sorta di alienazione dalla libertà, mancanza di una sua piena fruizione o mancato utilizzo della stessa a fin di bene, in modo tale da ampliare lo spazio. Ad unanima soggetta a costrizione il mondo appare inospitale, insignificante, se non addirittura nemico, ossia come un mondo caratterizzato da un deserto spirituale, forse persino da insensatezza, un crocevia.

Esiste comunque qualcosa in grado di salvare chi è in condizioni di schiavitù. È possibile una nuova apertura dello spazio. Tischner sostiene che attraverso Dio la libertà si estende, si libera con la forza della benevolenza e della verità. Allora la libertà "diviene nuovamente capacità di movimento e lo spazio si riempie di vie"12.

A questo punto Tischner si concentra sulla relazione personale fra uomo e Dio, sulla comunicazione interiore e sul legame spirituale con Colui che dà speranza e libertà vera. Occorre tuttavia aggiungere che sebbene nei Ritiri spirituali di Parigi non sia contenuto explicite un pensiero sul prossimo come possibile fondamento per un'apertura dello spazio, alla luce dell'intera opera di Tischner l'uomo possiede indubbiamente una grande forza e potere di cambiare l'altro, di accedere a tale spazio spirituale situato in fondo al prossimo. Questo spazio gli mostra un nuovo modo di vedere se stesso e il mondo, lo familiarizza con ciò che è bene e che nel bene amplia lo spazio della libertà. Condizioni indispensabili perché possa compiersi l'uscita dalla "ristrettezza" spirituale sono sia l'apertura ad accogliere la verità, sia il consenso a far accedere l'altro al proprio spazio spirituale. Solo allora diviene possibile una modifica anche dello spazio esterno. Questa trasformazione avviene sempre attraverso l'interiorità dell'uomo. Secondo Tischner tale direzione non è invertibile. Non ciò che è interno ha principio in ciò che è esterno ma avviene il contrario: "Ruscelli d'acqua viva scorrono a partire dall'interiorità dell'uomo"13. Tischner afferma che dalla qualità dello spazio interiore dell'uomo dipendono moltissime cose, soprattutto relazioni ordinate fra gli uomini basate sulla giustizia e sulla responsabilità nei confronti del prossimo. Soltanto esse sono in grado di rendere più umano lo spazio esterno. Sebbene Tischner sembri individuare il principio della libertà in ciò che è esterno, sembra anche che ispirazioni a ciò che è esterno possano anch'esse in qualche modo contribuire a rende più profondo lo spirito e a divenire un germe dell'uscita dell'uomo dalla ristrettezza di pensiero, di giudizio e di azione.

\footnotetext{
12 J. Tischner, Rekolekcje Paryskie, Znak, Kraków 2013, p. 87.

13 J. Tischner, Rekolekcje Paryskie, Znak, Kraków 2013, p. 88.
} 


\section{La libertà come "salute dell'anima"}

Le più significative analisi della libertà presenti nei Ritiri spirituali di Parigi si sviluppano attraverso la formula del "cuore libero" ripresa da Meister Ekhart. Tischner afferma che "ciascuno di noi ha una storia, ma la storia più importante è quella del proprio cuore" (290). Richiamandosi al mistico, teologo e filosofo medievale, Tischner valorizza l'affermazione secondo cui "un cuore libero può tutto". Il termine "tutto" compreso in questo enunciato radicalizza la sfera d'azione del cuore. Esso infatti può tutto se libero, ma non può tutto se costretto. La parola "libero" designa qui la condizione necessaria affinché si possa dare una definizione di ciò che è indispensabile al cuore e al suo agire. Se un cuore può tutto solo se libero, un cuore schiavo non può molto o addirittura non può nulla. Infatti esso deve prima aprirsi un varco verso la libertà e solo in seguito liberare la propria capacità di agire.

Tischner effettua una distinzione fra cuore e coscienza. La coscienza è comunque una voce che aiuta, prima ancora di compiere una scelta, a prendere una decisione, e che in seguito giudica il grado di conformità dell'azione dell'uomo a ciò che questi abbia identificato con il bene. Invece il cuore è uno spazio dinamico dellelemento del bene e del male: "anche il cuore ha un proprio ambiente nel quale batte, nel quale si sviluppa; tale ambiente del cuore è un bene, un segreto bene. Il cuore batte proprio là, più vivace, più forte, quando sente di poter essere buono"14.

Affrontando le considerazioni di Tischner si può avere l'impressione che questo filosofo, discutendo la formula ekhartiana, si sia servito più o meno consapevolmente di una parafrasi della distinzione berliniana fra libertà negativa "da" e libertà positiva "a". Ciò è confermato soprattutto sia dal susseguirsi delle analisi (prima egli svela le definizioni negative del cuore, poi perviene a quelle positive), sia dal designare la via che porta al cuore libero, che solamente in quanto tale può de facto tutto ${ }^{15}$.

14 J. Tischner, Rekolekcje Paryskie, Znak, Kraków 2013, p. 291.

${ }^{15}$ La caratterizzazione del cuore libero dovrebbe essere integrata con almeno alcune importanti questioni come: il problema della purezza del cuore, dell'amore, della dedizione e del sacrificio, che Tischner non affronta in questa pubblicazione ma alle quali egli accentra l'attenzione in molti altri luoghi della sua opera. In Miłości niemiłowanej [Amore non amato] il filosofo sviluppa il nodo tematico del cuore puro e del suo rapporto con la libertà dell'uomo (Wyd. S. Grotomirskiego, Kraków 1993); in Miłość nas rozumie [L’amore ci capisce] Tischner sottolinea le due tendenze più importanti presenti nell'amore nel contesto della libertà (Wyd. Znak, Kraków 2000). 
La scelta di definizioni negative del cuore conduce Tischner a mostrarne l'essenza. Egli tratta prima del cuore adescato (ingannato), poi del cuore vincolato (schiavo della paura di essere buono) infine del cuore avaro (alla ricerca del proprio).

Esiste un legame molto forte fra il cuore da una lato e la libertà dell'uomo nonché l’adeguata realizzazione di tale libertà dall'altro. Poiché per Tischner ha un'importanza fondamentale un uso adeguato della libertà, orientato quindi in una buona direzione, verso il bene, e predisposto alla scelta del bene, il cuore libero deve essere semplicemente libero "da" legocentrismo, da svariati travisamenti, da tentativi di impossessamento e dal male, perseguendo così la scelta del bene e "vivendo" di esso.

Nel primo significato qui affrontato, il "cuore libero" è un cuore che non è mai stato adescato e che non si è mai fatto ingannare. Costituirebbe pertanto una forza sui generis, un respingimento dell'attacco intentato dal male, uno strenuo difensore del bene grazie alla libertà di cui gode. L'adescamento è qui una specie di inganno, di raggiro compiuto nei confronti del cuore. Un cuore adescato è come isterilito per aver aderito alle illusioni e al falso, chiuso alla verità. Se gli si mente si provoca in esso uno stato di disorientamento. Nondimeno, esso non si rende conto inizialmente di tale disorientamento poiché l'errare, il vagare è divenuto il suo modo di procedere. Adescare significa designare il male come bene, convincere che il bene è proprio tale e non altro. Adescare significa anche dimostrare che proprio tale è il vero bene, e pertanto presentare argomenti a sostegno del fatto che non occorre pensare ad altro in quanto ogni eventuale alternativa risulterebbe inadeguata. Un cuore adescato crede alle illusioni e frena per un certo tempo la riflessione. Paradossalmente tuttavia, la scoperta del falso e della menzogna può avvenire nel cuore proprio grazie alla libertà, risvegliando in se stessi la volontà di discernimento, di un soliloquio profondo, pertanto attraverso l'apertura ad una riflessione intellettuale e all'analisi di quanto sia stato erroneamente riconosciuto come verità. Alla base di un buon "funzionamento" del cuore giace il dramma della volontà. Infatti, dopo aver scoperto la menzogna, il cuore ferito può non rialzarsi del tutto permanendo in una sorta di schiavitù, privo di fiducia e di speranza. Può restare come intrappolato in quella scoperta: si è chiaramente liberato dallerrare impostogli ma, ferito, non è in grado di liberarsi del tutto di questa esperienza. Chiudendosi, dunque, intende in qualche modo giustificare la propria ferita e precipita nella nicchia di una schiavitù certa.

Un secondo modo di intendere la realtà del "cuore libero" è sotto forma di disinvoltura ossia di liberazione. L’imbarazzo o impaccio è una sorta di catena, 
di costrizione. Tischner non colloca a tal proposito la disinvoltura o liberazione nel contesto del male, tuttavia parla di una certa paura percepita dall'uomo di non riuscire a trovare in sé le forze sufficienti per compiere il bene. Si tratta di un tipo specifico di paura che paralizza il libero agire del cuore, il quale vuole essere buono e affermare il bene. Questa paura è una specie di trattenersi dall'avere fiducia in quel cuore che intuisce il bene, che coglie come d' "istinto" il bene. Tischner scrive: "Oggi dobbiamo perdonare, domani dovremo dare acqua, cibo, e dopodomani, forse, morire per il prossimo. Temiamo che se saremo buoni una volta la logica del bene ci travolgerà fino a farci perire"16.

Esiste nelluomo la paura di esigenze del cuore sempre nuove che egli teme di non riuscire a gestire e talvolta, presentendole, se ne difende. È come dare per scontato che non resisteremo alle svariate prove che ci saranno sottoposte, quindi per sicurezza non agiamo, tratteniamo il movimento della volontà, diveniamo passivi.

Un terzo aspetto del cuore su quale Tischner si sofferma è la "ricerca del proprio", dunque il limitarsi del cuore a ciò che gli sembra a lui più vicino. Esso pertanto non si apre a quanto sia ben più vasto di lui, nonostante avverta spesso tale vastità. Tischner impiega a tal proposito il concetto di cuore "avaro", concentrato sulla propria piccolezza, accidentalità, finitezza, presenza qui ed ora. Un cuore "avaro" non sa essere generoso, dedito, devoto. L'avarizia del cuore è caratterizzata da una certa passività e forse persino da staticità in rapporto a ciò che è il suo dinamismo originario, e secondo Tischner la dinamica del cuore è tale ed è "progettata" perché esso possa avanzare sempre più in alto ${ }^{17}$. Un cuore avaro non ha alcun interesse ad avanzare verso l'alto. Esso rimane ad un certo livello, come immobile, esiste in virtù di una sorta di status quo in cui si sente "bene" e che gli basta. Se un cuore come questo non tende verso l'alto può invece aprire l'uomo a ciò che è basso, molto più basso di lui, provocandone l'abiezione, la distruzione, la messa in dubbio.

L'avarizia del cuore consisterebbe nell'assumere uno sguardo più ampio su unaltra prospettiva e nel trovare consolazione in ciò che è "su misura del suo cuore finito, del suo cuore piccolo e dolorosamente concentrato sulla propria piccolezza" ${ }^{\prime 1}$.

\footnotetext{
16 J. Tischner, Rekolekcje Paryskie, Znak, Kraków 2013, p. 293.

17 J. Tischner, Rekolekcje Paryskie, Znak, Kraków 2013, p. 293.

18 J. Tischner, Rekolekcje Paryskie, Znak, Kraków 2013, p. 293.
} 
Tischner sintetizza le tre varianti negative del cuore di cui si è detto sopra nella definizione di "malattia dell'anima". In un cuore riluttante, vuoto, costretto, esiste un'enorme lacuna che potrebbe essere colmata da Dio, ma ciò non può compiersi se ci si chiude ad esso. Finché il cuore procederà a ritroso o resterà immobile, esso non vorrà accogliere in sé la prospettiva di un'apertura attraverso la libertà.

Come, pertanto, può avvenire l'apertura di un cuore che ha comunque la possibilità di entrare nell'ottica di un nuovo movimento? Tischner non sorprende con la sua risposta affermano che ciò potrebbe avvenire attraverso l'amore, ma sorprende quando afferma che tale apertura può accadere anche attraverso l'obbedienza. Soltanto attraverso il fenomeno dell'obbedienza (e pertanto ascoltando da un lato ciò che ci viene detto dal prossimo, anche da Dio, e dall'altro noi stessi) è possibile parlare di cuore libero. Il paradosso rappresentato dalla relazione libertà-obbedienza riflette ciò che in un cuore libero si manifesta come un movimento positivo di apertura al prossimo, al bene, alla realizzazione di se stessi. Tischner constata: "Obbedire significa ascoltare. Significa ascoltare il prossimo ascoltando al contempo se stessi, ascoltare il prossimo che ridesta il nostro cuore dal sonno e ascoltare il proprio cuore, come un uccello che batte le ali ritrovandosi improvvisamente nel proprio elemento" 19 .

Tischner radicalizza e restringe la tesi del cuore libero affermando che l'atto di incontrare Dio comporta che l'uomo abbia in sé un cuore davvero libero in grado di potere ogni cosa e di riuscire in tutto, un cuore né avaro, né illuso, che neppure cerca il proprio ma che vuole dare se stesso, persino abbandonarsi completamente all'Altro. In altre parole lo spazio in cui hanno luogo il sentire e l'ascoltare l'invito, il richiamo, l'ammonimento, l'avvertimento è il cuore umano, ma soltanto un cuore libero può ascoltare Dio e il richiamo del proprio fratello. Esiste la necessità di percepire, di udire e di ascoltare Dio e l'uomo, perché attraverso l'ascolto si perviene all'invito: l'invito al bene e l'avvertimento volto a proteggere dal male. Tischner ritiene che "l'ascolto di una voce segna l'inizio, il momento in cui l'umanità si ridesta nell'uomo" ${ }^{20}$. Se abbia avuto o avrà luogo un indebolimento dell'ascolto, ciò è avvenuto o avverrà a causa della libertà dell'uomo. Quando l'uomo non ha intenzione di ascoltare il prossimo e Dio, soffoca una voce risuonata in lui o non percepisce più alcun suono per

19 J. Tischner, Rekolekcje Paryskie, Znak, Kraków 2013, p. 296.

${ }^{20}$ J. Tischner, Rekolekcje Paryskie, Znak, Kraków 2013, p. 288. 
aver rinunciato già da tempo all'ascolto. In tali circostanze egli diviene un uomo di "cattivo cuore" perennemente "concentrato, per questi o altri motivi, esclusivamente su se stesso. E questo uomo dal cuore chiuso non toccherà mai Dio, che è il Dio del bene, che è il Dio della massima bontà" ${ }^{21}$.

I tre diversi modi di concepire la libertà sopra esposti, ossia come scelta, come spazio e in relazione con il cuore umano, mostrano la drammaticità di questa condizione, ma anche la libertà come proprietà strutturale del soggetto. Infatti il coinvolgimento della libertà dell'uomo nel dramma del bene e del male non vanifica la possibilità di un'assunzione autonoma di una decisione da parte sua.

Quantunque nel ciclo di conferenze tischneriane di cui qui si tratta prevalga la tendenza a soffermare l'attenzione su nodi tematici legati alla vita interiore dell'uomo (soprattutto alla relazione uomo-Dio) nonché sulla preoccupazione per la Polonia, una patria che negli anni ' 80 del secolo scorso ebbe bisogno di speranza, verità e libertà, le questioni filosofiche descritte, presenti nel ciclo, possono per un certo senso divenire origine di un più ampio dibattito sulla natura della libertà, che fino alla fine della propria produzione Tischner ha svolto nelle pagine delle sue pubblicazioni.

Ciò che Tischner ha qui segnalato necessita chiaramente di ulteriori sviluppi e lascia al lettore una serie di domande che accrescono tanto più il desiderio di approfondimento quanto meno il filosofo abbia dato ad esse una risposta definitiva. La formula concisa della conferenza ha certamente impedito a Tischner di calarsi ad un livello più profondo delle analisi fenomenologiche, ma sembra che egli abbia lasciato agli uditori uno spiraglio che possa permettergli di approdare ad una più profonda comprensione della libertà rispetto ad altri e a se stesso. La medesima formula ha saputo al contempo ispirare a proseguire la ricerca e ad affrontare la scoperta di altri sensi della libertà umana.

\section{Bibliography}

Tischner J., Miłość niemiłowana, Wyd. S. Grotomirskiego, Kraków 1993.

Tischner J., Rekolekcje Paryskie, Znak, Kraków 2013.

Tischner J., Zarys filozofii człowieka dla duszpasterzy i artystów, in: „Myślenie w żywiole piękna", Znak, Kraków 2004.

${ }^{21}$ J. Tischner, Rekolekcje Paryskie, Znak, Kraków 2013, p. 296. 